\title{
Switching-based Sinusoidal Disturbance Rejection for Uncertain Stable Linear Systems
}

\author{
Yang Wang, Gilberto Pin, Andrea Serrani and Thomas Parisini
}

\begin{abstract}
The problem of rejection of sinusoidal disturbances with known frequencies acting on an unknown singleinput single-output linear system is addressed in this note. We present a new approach that does not require knowledge of the frequency response of the transfer function over the frequency of interest. The proposed methodology reposes upon the combination of the classic feedforward control algorithm and logic-based switching. The use of three different switching logics is proposed in this paper, namely: pre-routed, dwell-time and hysteresis switching. A comparative evaluation of the three switching strategies is performed via a simulation study.
\end{abstract}

\section{INTRODUCTION AND PROBLEM FORMULATION}

Rejection of harmonic disturbances occurring in a control system remains a central theme in control, fueled by a large number of technological applications, from vibration suppression [1] to active noise control [2], to wave attenuation in marine systems [3]. A common thread across the different approaches pursued by the control community to the solution of various manifestations of the harmonic disturbance rejection problem is the ubiquitous internal model principle [4], which prescribes that a suitable copy of the system generating the disturbance must be embedded in the controller to ensure robust regulation in the presence of model uncertainties. While the harmonic disturbance rejection problem is subsumed by the more general output regulation problem [5], in its prototypical form for SISO LTI plant models, the former problem is cast into the following setup

$$
\begin{aligned}
& \dot{x}=A(\mu) x+B(\mu)[\hat{d}(t)-d(t)], \quad x(0)=x_{0} \in \mathbb{R}^{r} \\
& y=C(\mu) x
\end{aligned}
$$

where (1) is an $r$-dimensional realization of the internally stable interconnection of an uncertain plant model and a robust stabilizer. System (1) is driven by the difference between a sinusoid of known frequency, $\omega^{\star} \in \mathbb{R}_{>0}$

$$
d(t)=\psi_{1} \cos \left(\omega^{\star} t\right)+\psi_{2} \sin \left(\omega^{\star} t\right)
$$

and an estimate of the disturbance, $\hat{d}(t) \in \mathbb{R}$, generated by a feedforward regulator. The vectors $\mu \in \mathbb{R}^{p}$ and $\psi:=$ $\operatorname{col}\left(\psi_{1}, \psi_{2}\right) \in \mathbb{R}^{2}$ collect the uncertain parameters of the plant model and the disturbance, respectively. It is assumed that $\mu$ ranges on a given known compact set, $\mathcal{P} \subset \mathbb{R}^{p}$. For future use, we let $W_{\mu}(s):=C(\mu)(s I-A(\mu))^{-1} B(\mu)$ denote

Yang Wang and Thomas Parisini are with the Department of Electrical and Electronic Engineering, Imperial College, London UK. Gilberto Pin is with Electrolux Professional, S.p.A., Italy; Andrea Serrani is with the Department of Electrical and Computer Engineering, The Ohio State University, Columbus $\mathrm{OH}$ - USA. Corresponding author: A. Serrani, serrani.1@osu.edu the transfer function of system (1). System (1) is assumed to be internally stable, robustly with respect to $\mu \in \mathcal{P}$ :

Assumption 1.1: There exist constants $a_{1}, a_{2}>0$ such that the parameterized family $P_{x}: \mathbb{R}^{p} \rightarrow \mathbb{R}^{r \times r}$ of solutions of the Lyapunov equation $P_{x}(\mu) A(\mu)+A^{T}(\mu) P_{x}(\mu)=-I$ satisfies $a_{1} I \leq P_{x}(\mu) \leq a_{2} I$ for all $\mu \in \mathcal{P}$.

The sinusoidal disturbance in (2) can be represented as the output of an exosystem of the form

$$
\begin{aligned}
& \dot{\omega}=S \omega, \quad \omega(0)=\omega_{0}=\psi \in \mathbb{R}^{2} \\
& d=\Gamma \omega
\end{aligned}
$$

where $S=\left(\begin{array}{cc}0 & \omega^{\star} \\ -\omega^{\star} & 0\end{array}\right), \quad \Gamma=\left(\begin{array}{ll}1 & 0\end{array}\right)$. As a consequence, the original problem is cast into the framework of output regulation [4], [6], with the additional assumption of internal stability of the plant model:

Problem 1: (Output Regulation Problem) For system (1), design a dynamic output-feedback controller of the form

$$
\begin{aligned}
& \dot{\xi}=f_{a}(\xi, y), \quad \xi(0)=\xi_{0} \in \mathbb{R}^{m} \\
& \hat{d}=h_{a}(\xi, y),
\end{aligned}
$$

with $f_{a}(\cdot, \cdot): \mathbb{R}^{m} \times \mathbb{R} \mapsto \mathbb{R}^{m}$ and $h_{a}(\cdot, \cdot): \mathbb{R}^{m} \times \mathbb{R} \mapsto \mathbb{R}$, such that the trajectories of the closed-loop system (1), (3) and (4) originating from all initial conditions $x_{0} \in \mathbb{R}^{r}, \omega_{0} \in$ $\mathbb{R}^{2}$ and $\xi_{0} \in \mathcal{X}$, where $\mathcal{X} \subset \mathbb{R}^{m}$ is a set to be determined, are bounded and satisfy $\lim _{t \rightarrow \infty} y(t)=0$ for all $\mu \in \mathcal{P}$. $\triangleleft$

Necessary and sufficient conditions of solution of Problem 1 have been known for a long time [5]-[7]. Specialized to the case of SISO systems, these conditions are hereby assumed:

Assumption 1.2: (i) System (1) is controllable and observable for any $\mu \in \mathcal{P}$; (ii) Over the range of frequencies of interest, $W_{\mu}\left(j \omega^{\star}\right) \neq 0$ for all $\mu \in \mathcal{P}$.

Early work in the realm of adaptive feedforward control (AFC) assume knowledge of $\operatorname{Re}\left\{W_{\mu}\left(j \omega_{\star}\right)\right\}$ and $\operatorname{Im}\left\{W_{\mu}\left(j \omega_{\star}\right)\right\}$ as a prerequisite for the controller design [1], [8]-[10], such a condition is usually termed an SPR-like condition. Subsequent works have attempted an adaptive estimation of said quantities within AFC schemes [11], [12]; however, issues related to asymptotic convergence and interaction with the plant dynamics were left open. The recent contribution [13], has shown that given the knowledge of either sign $\operatorname{Re}\left\{W_{\mu}\left(j \omega_{\star}\right)\right\}$ or sign $\operatorname{Im}\left\{W_{\mu}\left(j \omega_{\star}\right)\right\}$, one or both of the two following dynamic feedback controllers with $\hat{d}=\hat{\omega}_{1}$ solves the Problem 1 for sufficient small values of 


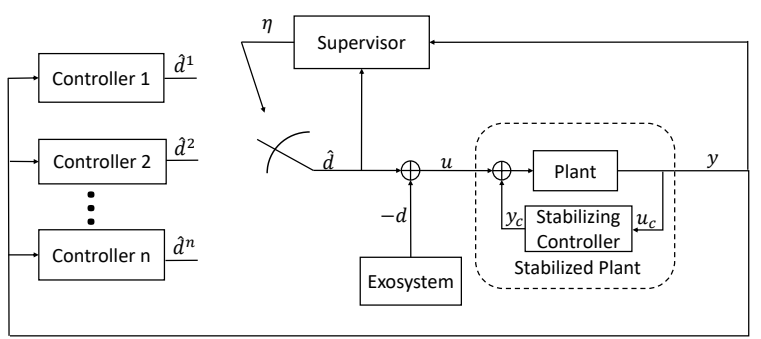

Fig. 1. Multi-Controller set up for output regulation problem.

the controller gain $k>0$ :

$$
\begin{aligned}
& \left\{\begin{array}{l}
\dot{\hat{\omega}}_{1}=\omega^{\star} \hat{\omega}_{2}-k \operatorname{sign} \operatorname{Re}\left\{W_{\mu}\left(j \omega^{\star}\right)\right\} y \\
\dot{\hat{\omega}}_{2}=-\omega^{\star} \hat{\omega}_{1}
\end{array}\right. \\
& \left\{\begin{array}{l}
\dot{\hat{\omega}}_{1}=\omega^{\star} \hat{\omega}_{2} \\
\dot{\hat{\omega}}_{2}=-\omega^{\star} \hat{\omega}_{1}-k \operatorname{sign} \operatorname{Im}\left\{W_{\mu}\left(j \omega^{\star}\right)\right\} y
\end{array}\right.
\end{aligned}
$$

In [14], the authors have proposed an adaptive control scheme that completely removes the necessity of knowing a priori the sign of the real and imaginary parts of $W_{\mu}\left(j \omega^{\star}\right)$. The controller in [14] is characterized by a relatively high dimensionality, due to the necessity to employ a multiple model adaptive control scheme. In this paper, we propose an alternative design, where multiple-model adaptive techniques are replaced by switching mechanisms among fixed controllers. Several different switching strategies are proposed, within a common baseline control architecture. It is shown that the proposed approach removes some of the pitfalls of the multiple-model adaptive controller of [14], albeit still requiring a relatively large-dimensional state space for the overall controller.

The paper is organized as follows: The structure of the multi-controller employed in this paper is given in Section II. In Section III, a brief overview of the switching strategies relevant to the approach pursued in this paper is presented, based on the lucid exposition found in [15]. The analysis of the proposed design is presented in Section IV, whereas a comparative simulation study is reported in Section V. Concluding remarks are offered in Section VI.

Notation: We denote with $\|\cdot\|$ both the Euclidean vector norm and the corresponding induced matrix norm.

\section{State-Sharing Multi-Controller}

The overall control architecture, depicted in Fig.1, follows the general paradigm of state-shared multi-controllers proposed by Morse [15] [16]. The control signal applied to the plant is $\hat{d}(t):=\hat{d}^{\eta(t)}(t)$, where $\eta:[0, \infty) \mapsto \mathcal{I}$ is a piecewise-constant switching signal taking values in the index set of the family of the candidate controllers $\mathcal{I}:=$ $\{1,2, \cdots, n\}$. The system that generates the switching signal is referred to as the supervisory system. In the architecture of Fig. 1, the candidate controllers $C^{i}, i \in \mathcal{I}$, are selected as

$$
\begin{aligned}
\dot{\hat{w}}^{i} & =S \hat{w}^{i}-k \hat{\vartheta}^{i} y, \quad \hat{w}^{i}(0)=\hat{w}_{0}^{i} \\
\hat{d}^{i} & =\Gamma \hat{w}^{i}, \quad i \in \mathcal{I}
\end{aligned}
$$

where $\hat{\vartheta}^{i}$ is a constant estimate of the plant response at the frequency of excitation, $\vartheta(\mu):=\left(\begin{array}{ll}\vartheta_{1} & \vartheta_{2}\end{array}\right)^{T}=$ $\left(\operatorname{Re}\left\{W_{\mu}\left(j \omega^{\star}\right)\right\} \operatorname{Im}\left\{W_{\mu}\left(j \omega^{\star}\right)\right\}\right)^{T}$. The parameter $\vartheta \in \mathbb{R}^{2}$ is unknown, but assumed to range in a known set. Specifically, let the set $\Theta \subset \mathbb{R}^{2}$ be the annular region defined, for given real numbers $0<\delta_{1}<\delta_{2}$, as

$$
\Theta:=\left\{\vartheta \in \mathbb{R}^{2} \mid \delta_{1}^{2} \leq \vartheta_{1}^{2}+\vartheta_{2}^{2} \leq \delta_{2}^{2}\right\}
$$

and consider the following assumption:

Assumption 2.1: The unknown parameter vector $\vartheta(\mu)$ satisfies $\vartheta(\mu) \in \operatorname{int} \Theta$ for all $\mu \in \mathcal{P}$.

It is noted that the set $\Theta$ is not convex. The family of the candidate controllers is designed in such a way that $\hat{\vartheta}^{i} \in \Theta$ for all $i \in \mathcal{I}$, and there exists at least one controller $C^{j}, j \in \mathcal{I}$ with parameter estimate $\hat{\vartheta}^{j}$ satisfying

$$
\left\|\hat{\vartheta}^{j}-\vartheta\right\| \leq \rho,
$$

where $\rho$ is a constant to be selected. In the following sections, it will be shown that, for an appropriate choice of $\rho$ and $k$, (9) ensures that there exists a subset of the family of the candidate controllers, with index set denoted by $\mathcal{I}^{\star}$ that solves the output regulation problem for any $\mu \in \mathcal{P}$.

To reduce the dimension of the overall controller, a different parameterization of the controller is first employed by way of the coordinate change $\hat{w}_{c}^{i}:=T_{c}^{i} \hat{w}^{i}, i \in \mathcal{I}$, where

$$
T_{c}^{i}:=\frac{1}{\left\|\hat{\vartheta}^{i}\right\|}\left(\begin{array}{cc}
\hat{\vartheta}_{1}^{i} & -\hat{\vartheta}_{2}^{i} \\
\hat{\vartheta}_{2}^{i} & \hat{\vartheta}_{1}^{i}
\end{array}\right)
$$

and $\hat{\vartheta}_{j}^{i}, j=1,2$ represents the $j$-th element of the vector $\hat{\vartheta}^{i}$. This yields the set of state-sharing controllers [15] (equivalent to (7))

$$
\begin{aligned}
\dot{\hat{w}}_{c} & =S \hat{w}_{c}-k G_{c} y, \quad \hat{w}_{c}(0)=\hat{w}_{c 0} \in \mathbb{R}^{2} \\
\hat{d}^{i} & =\hat{\theta}^{i T} \hat{w}_{c}, \quad i \in \mathcal{I}
\end{aligned}
$$

with $G_{c}=\Gamma^{T}$ and $\hat{\theta}^{i}=\left(\hat{\vartheta}_{1}^{i}-\hat{\vartheta}_{2}^{i}\right)^{T}$. Since each member of (10) has the same structure of either (5) or (6), the existence of a controller that solves the problem is guaranteed for a suitable value of $k$. Also, note that (10) reduces the dimension of the controller from $3 n$ to $2+n$ for a single frequency. Clearly, Assumption 2.1 holds for the reparameterized vector $\theta:=\left(\hat{\vartheta}_{1}^{i}-\hat{\vartheta}_{2}^{i}\right)^{T}$, whereas condition (9) needs to be revised for the re-parameterized estimates $\hat{\theta}^{i}$.

Remark 2.1: While the multi-controller (10) is used for the implementation of the algorithm, we sometimes refer to its equivalent form for the ease of analysis (7).

The control problem is thus reformulated as follows:

Problem 2: (Switching Logic Design Problem) For system (1), (3) and (10), design a supervisor that generates a suitable switching signal $\eta$ with a selection for $k>0$ and $\rho>0$ such that trajectories of the closed-loop system originating from any $x_{0} \in \mathbb{R}^{r}, \omega_{0} \in \mathbb{R}^{2}$ and $\hat{\omega}_{c 0} \in \mathbb{R}^{2}$ are bounded and satisfy $\lim _{t \rightarrow \infty} y(t)=0$ for all $\mu \in \mathcal{P}$. $\triangleleft$ 


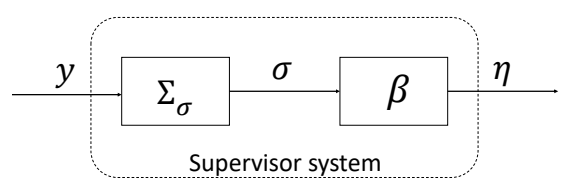

Fig. 2. Non-estimator based supervisor.

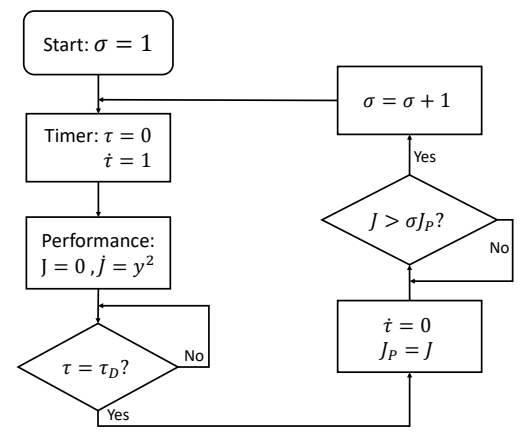

Fig. 3. Flowchart of the scheduling function $\Sigma_{\sigma}$ in Fig. 2

\section{SwITCHING LogIC}

This section presents the development of the supervisor system in Fig. 1 by using three different switching logics: Pre-routed, Hysteresis and Dwell-time switching [15], [17].

\section{A. Non-estimator-based Supervisor: Pre-routed Switching}

The non-estimator-based supervisor is the cascade connection of a scheduling logic $\Sigma_{\sigma}$ and a routing function $\beta(\cdot):\{1,2,3, \cdots,+\infty\} \mapsto \mathcal{I}$, as shown in Fig. 2. The output $\sigma(\cdot):[0,+\infty) \mapsto \mathbb{Z}^{+}$is a piecewise-constant signal to be determined. The routing function $\beta(\cdot)$ is constructed to fulfill the following revisitation property [15]:

Property 3.1 (Revisitation Property): For any $q \in \mathcal{I}$ and any $i \in \mathbb{N}$, there exists an integer $j \geq i$ at which $\beta(j)=q$.

Essentially, along the pre-routed path $\beta(\sigma)$ each candidate controller is revisited infinitely often. Here, we define $\beta(\sigma):=\bmod (\sigma, n)+1$. The flow chart of the scheduling logic $\Sigma_{\sigma}$ is given in Fig. 3, where $\tau_{D}$ is a predefined time constant. During the first $\tau_{D}$ time units after a switch is initiated, $\tau$ is increased form 0 to $\tau_{D}$, while $J(t)$ evolves according to

$$
\dot{J}(t)=(y(t))^{2} .
$$

At the end of this interval of time, the timer $\tau$ is turned off and $J_{P}$ is set to be equal to the present value of $J$. So long as $J(t)$ remains smaller or equal than $\sigma J_{P}, J(t)$ is updated continuously according to (11). If and when $J(t)>\sigma J_{P}, \sigma$ is incremented by 1 , meanwhile $\tau, J$ and $J_{P}$ are set to zero, and the entire process is repeated. Note that, thanks to $\tau_{D}$, infinitely fast switching is avoided and the solutions to the differential equations involved exist and are unique.

\section{B. Estimator-based Supervisor}

Alternative switching strategies considered herein (hysteresis and dwell-time) require a multi-estimator controller architecture as shown in Fig. 4. In this scheme, $\Sigma_{S}$ represents the switching logic, whose function is to determine $\eta$ based

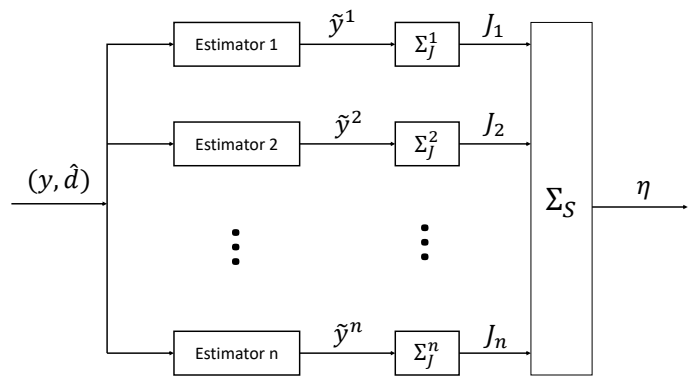

Fig. 4. Estimator-based supervisor system.

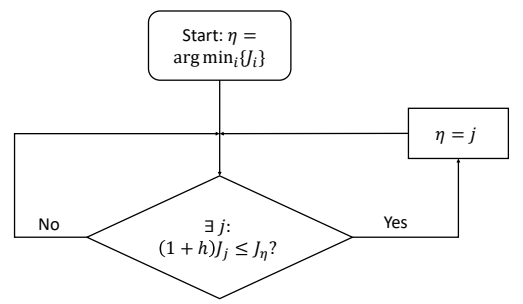

Fig. 5. Flowchart of the hysteresis switching logic.

on $J_{i}, i \in \mathcal{I}$. Following [14], let $\Pi(\mu) \in \mathbb{R}^{r \times 2}$ be the unique solution of the Sylvester equation $\Pi(\mu) S=A(\mu) \Pi(\mu)+$ $B(\mu) \Gamma$ and change coordinates in (1) and (7) as $\zeta:=\hat{w}^{\eta}-w$ and $z:=x-\Pi(\mu) \zeta$ to obtain

$$
\begin{aligned}
& \dot{z}=A(\mu) z+k \Pi(\mu) \hat{\vartheta}^{\eta} y, \quad z(0)=z_{0} \in \mathbb{R}^{r} \\
& \dot{\zeta}=S \zeta-k \hat{\vartheta}^{\eta} y, \quad \zeta(0)=\zeta_{0} \in \mathbb{R}^{2} \\
& y=C(\mu) z+\vartheta^{T}(\mu) \zeta,
\end{aligned}
$$

where $\vartheta^{T}(\mu)=C(\mu) \Pi(\mu)$. To define the performance index for each candidate controller, we design the adaptive observer

$$
\begin{aligned}
\dot{\hat{\zeta}} & =S \hat{\zeta}-k \hat{\vartheta}^{\eta} y-\varepsilon \hat{\vartheta}^{\eta}\left(\hat{\vartheta}^{\eta T} \hat{\zeta}-y\right), \quad \hat{\zeta}(0)=\hat{\zeta}_{0} \in \mathbb{R}^{2} \\
\hat{y}^{i} & =\hat{\vartheta}^{i T} \hat{\zeta}, \quad i \in \mathcal{I}
\end{aligned}
$$

where $\varepsilon \in \mathbb{R}_{>0}$. For each $i \in \mathcal{I}$, the performance signal generator $\Sigma_{J}^{i}$ is defined as $\dot{J}_{i}=-\lambda J_{i}+\left(\tilde{y}^{i}\right)^{2}$, with $J_{i}(0)=$ $J_{i 0}, \tilde{y}:=\hat{y}^{i}-y$ and $\lambda \in \mathbb{R}_{>0}$ is a forgetting factor. Given $J_{i}$, the design of the supervisor system is completed by the selection of a suitable switching logic $\Sigma_{S}$. In what follows, we give a brief account of the two switching strategies.

1) Hysteresis Switching [17]: The mechanism behind hysteresis switching is shown in Fig. 5. Let $h \in \mathbb{R}_{>0}$, called the hysteresis constant, be given. Assume that that at certain time $t_{n}$, the value of $\eta\left(t_{n}\right)$ switches to some $j \in \mathcal{I}$. Then the value of $\eta$ is kept constant until the time $t_{n+1} \geq t_{n}$ when $(1+h) \min _{i \in \mathcal{I}}\left\{\left(J_{i}\left(t_{n+1}\right)\right\}<J_{j}\left(t_{n+1}\right)\right.$, at which point $\eta\left(t_{n+1}\right)$ is set as $\eta\left(t_{n+1}\right)=\arg \min _{i \in \mathcal{I}}\left\{J_{i}\left(t_{n+1}\right)\right\}$. Repeating the above steps, a piecewise-constant signal $\eta$ is generated, which is continuous from the right. The selection $J_{i 0}>0$ avoids infinitely fast switching in the initialization of the algorithm. Possible non-uniqueness in the selection of $\arg \min _{i \in \mathcal{I}}\left\{J_{i}\left(t_{n+1}\right)\right\}$ is resolved by an arbitrary assignment among the available choices. 


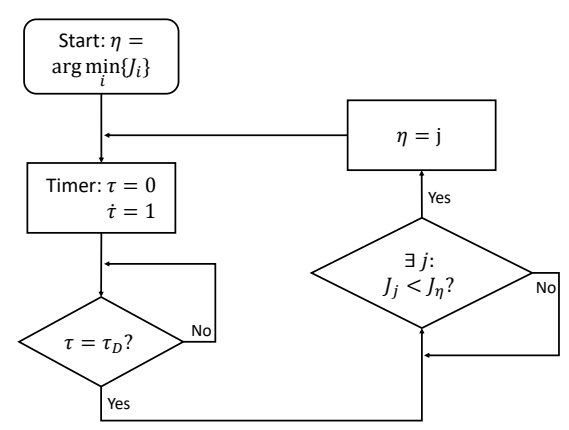

Fig. 6. Flowchart of the Dwell-time switching logic.

2) Dwell Time Switching [18]: the mechanism is illustrated in Fig.6. Let $\tau_{D}>0$ be a chosen dwell-time constant, and assume that at a time $t_{n}, \eta$ switches to some $j \in \mathcal{I}$. The value of $\eta$ is then kept constant until a time $t_{n+1} \geq t_{n}$ occurs such that $t_{n+1}-t_{n} \geq \tau_{D}$ and $\min _{i \in \mathcal{I}}\left\{\left(J_{i}\left(t_{n+1}\right)\right\}<J_{j}\left(t_{n+1}\right)\right.$. At that point, $\eta\left(t_{n+1}\right)$ is set as $\eta\left(t_{n+1}\right)=\arg \min _{i \in \mathcal{I}}\left\{J_{i}\left(t_{n+1}\right)\right\}$. Similarly to the hysteresis switching, when arg min is not unique, $\eta$ can be arbitrarily selected among those available choices. $\tau_{D}$ avoids the occurrence of infinitely fast switching.

\section{Stability Analysis}

In this section, we provide a concise treatment of the stability and convergence analysis for the multi-controller (10) (equivalently, (7)) for each of the considered switching logic. To begin, we establish properties of a certain Lyapunov function candidate that are instrumental in the ensuing analysis. Details are given in [14].

Property 4.1: There exist a scalar $\bar{k}>0$ and constants $0<c_{1}<c_{2} \leq c_{3}$ such that the solution $P_{o}:(\vartheta, k) \mapsto \mathbb{R}^{2 \times 2}$ of the parameterized family of Lyapunov equations

$$
\left.P_{o}\left[S-k \vartheta \vartheta^{T}\right]+\left[S-k \vartheta \vartheta^{T}\right]^{T} P_{o} k\right)=-k \vartheta^{T} \vartheta I
$$

satisfies $c_{1} I \leq P_{o}(\vartheta, k) \leq c_{2} I$ and $\left\|P_{o}(\vartheta, k)\right\| \leq c_{3}$ for all $(\vartheta, k) \in \Theta \times[0, \bar{k}]$.

Let the time sequence $\left\{T_{n}\right\}_{n=1}^{N}$ denote the instants at which switching takes place, and consider in any time interval $t \in$ $\left[T_{n-1}, T_{n}\right)$ the LTV system (12), which can be seen as the feedback interconnection of the two linear systems :

$$
\begin{gathered}
\Sigma_{1}: \dot{\zeta}=\left[S-k \vartheta \vartheta^{T}\right] \zeta-k \tilde{\vartheta}^{\eta} \vartheta^{T} \zeta-k \hat{\vartheta}^{\eta} \nu_{1}, \quad y_{1}=\theta^{T} \zeta \\
\Sigma_{2}: \dot{z}=A z+k \Pi \hat{\theta}^{\eta} C z+k \Pi \hat{\theta}^{\eta} \nu_{2}, \quad y_{2}=C z
\end{gathered}
$$

with the interconnection structure $\nu_{1}=y_{2}, \nu_{2}=y_{1}$ and $\tilde{\vartheta}^{\eta}:=\hat{\vartheta}^{\eta}-\vartheta$. For each system $\Sigma_{i}, i=1,2$, the following intermediate results hold, due to the fact that there exists at least one candidate controller with $\hat{\theta}^{i}$ verifying (9):

Lemma 4.2: There exist scalars $\gamma_{1}^{\star}>0, \rho^{\star}>0$ and $k_{1}^{\star} \in$ $(0, \bar{k}]$ such that system $\Sigma_{1}$ is strictly dissipative with respect to the supply rate $q_{1}\left(\nu_{1}, \eta_{1}\right)=\gamma_{1}^{\star 2}\left|\nu_{1}\right|^{2}-\left|y_{1}\right|^{2}$ for all $k \in$ $\left(0, k_{1}^{\star}\right)$ and $\left\|\tilde{\vartheta}^{\eta}\right\| \leq \rho^{\star}$, with quadratic, positive definite and decrescent storage function $V_{1}(\zeta)=2 k^{-1} \zeta^{T} P_{o}(\theta, k) \zeta$.
Lemma 4.3: There exist scalars $\gamma_{2}^{\star}>0$ and $k_{2}^{\star} \in \mathbb{R}_{>0}$ such that $\Sigma_{2}$ is strictly dissipative with respect to the supply rate $q_{2}\left(\nu_{2}, \eta_{2}\right)=k^{2} \gamma_{2}^{\star 2}\left|\nu_{2}\right|^{2}-\left|y_{2}\right|^{2}$ for all $k \in\left(0, k_{2}^{\star}\right)$, with quadratic and positive definite storage function $V_{2}(z)=$ $z^{T} P_{x}(\mu) z$.

The proofs of the lemmas follow from elementary Lyapunov arguments. It is worth noticing that, since the interconnected system is globally Lipschitz uniformly in $t$, solutions exist uniquely on $[0, \infty)$.

\section{Non-estimator based controller}

Next, we establish the stability property of the overall system with the non-estimator based switching mechanism.

Theorem 4.4: Given system (1), there exist scalars $k^{\star} \in$ $\mathbb{R}_{>0}$ and $\rho^{\star} \in \mathbb{R}_{>0}$ such that, for all the state-sharing mulit controller (10) with $k \in\left(0, k^{\star}\right)$ and $\rho \in\left(0, \rho^{\star}\right)$, the nonestimator based supervisor system described in Section III-A solves the problem defined in Problem 2.

Proof: Applying Lemmas $4.2-4.3$ and combining the $\mathcal{L}_{2}$ gains of the single subsystems, Letting $k^{\star}:=$ $\min \left\{k_{1}^{\star}, k_{2}^{\star},\left(\gamma_{1}^{\star} \gamma_{2}^{\star}\right)^{-1}\right\}$, it follows that system (12) is a small-gain interconnection (with respect to the $\mathcal{L}_{2}$-norm) for all $k \in\left(0, k^{\star}\right)$. Since $\rho \in\left(0, \rho^{\star}\right)$, there exists a subset of the family of the candidate controllers, with index set denoted by $\mathcal{I}^{\star}$, whose members solve the output regulation problem. This implies that $y \in \mathcal{L}_{2}$ if $\eta \in \mathcal{I}^{\star}$. Assume that at $t=T_{m}$ $\eta(t)$ switches to some $q \in \mathcal{I}^{\star}$. As shown in [15, Section 4.2], there exists an integer $\bar{\sigma}(q)>0$, which depends only on the parameters of the controller, satisfying

$$
J(t)=\int_{T_{m}}^{t}(y(\tau))^{2} d \tau \leq \bar{\sigma}(q) \int_{T_{m}}^{T_{m}+\tau_{D}}(y(\tau))^{2} d \tau
$$

for all $t \in\left[T_{m}, T_{m+1}\right)$. Resorting to the revisitation Property 3.1 , and due to the fact that $\sigma$ will increment by 1 after each switch, there always exists an instant $T_{m}$ at which $\sigma\left(T_{m}\right)>\bar{\sigma}(q)$. This indicates that no more switching will occur beyond this point and $T_{m+1}=+\infty$. Consequently, $\lim _{t \rightarrow \infty} y(t)=0$.

\section{Estimator-based controllers}

For the estimator-based supervisor system, the dynamics of the observation error, $\tilde{\zeta}:=\hat{\zeta}-\zeta$, are given by

$$
\dot{\tilde{\zeta}}=\left(S-\varepsilon \hat{\vartheta}^{\eta} \hat{\vartheta}^{\eta T}\right) \tilde{\zeta}-\varepsilon \hat{\vartheta}^{\eta}\left(\tilde{\vartheta}^{\eta T} \zeta-C(\mu) z\right)
$$

Resorting to Property 4.1, one can verify that there exists a positive definite symmetric matrix-valued function $P_{e}:\left(\hat{\vartheta}^{\eta}, \varepsilon\right) \mapsto \mathbb{R}^{2 \times 2}$ solving the parametrized family of Lyapunov equations $P_{e}\left[S-\varepsilon \hat{\vartheta}^{\eta} \hat{\vartheta}^{\eta T}\right]+\left[S-\varepsilon \hat{\vartheta}^{\eta} \hat{\vartheta}^{\eta T}\right]^{T} P_{e}=$ $-\varepsilon \hat{\vartheta}^{\eta T} \hat{\vartheta}^{\eta} I$. Over any time interval $t \in\left[T_{n-1}, T_{n}\right)$, the overall system can be written in the following form

$$
\dot{\xi}=F\left(k, \varepsilon, \hat{\vartheta}^{\eta}\right) \xi+H \theta \tilde{u}^{\eta}, \quad y=C_{p} \xi
$$

with state $\xi:=\left(\begin{array}{lll}\zeta^{T} & z^{T} & \tilde{\zeta}^{T}\end{array}\right)^{T}$ and input error $\tilde{u}^{\eta}:=\tilde{\vartheta}^{\eta T} \zeta$. In (17), $H$ is a constant matrix of suitable dimension, and $C_{p}=\left[\vartheta^{T}, C(\mu), 0,0\right] \in \mathbb{R}^{r+4}$. Using arguments similar 

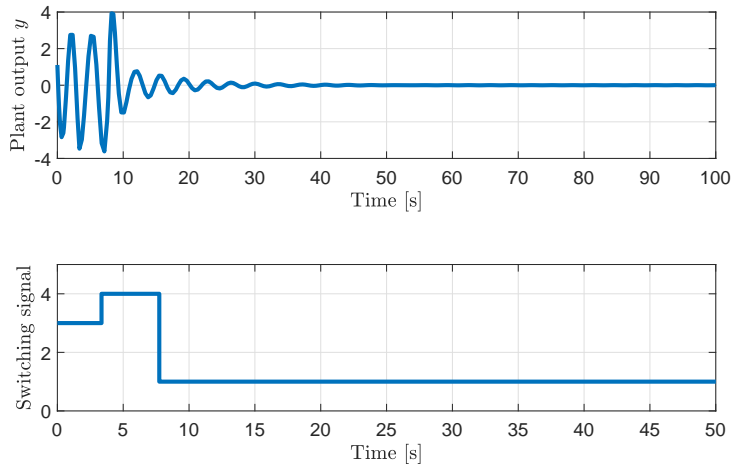

Fig. 7. System response using the pre-routed switching mechanism
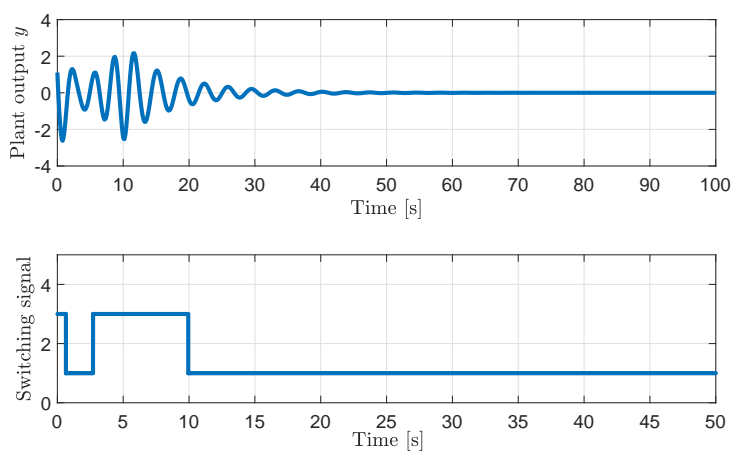

Fig. 8. System response using the hysteresis switching mechanism

to those employed in the proof of Theorem 4.4, it can be verified that $F\left(k, \varepsilon, \hat{\vartheta}^{\eta}\right)$ is a Hurwitz matrix for any $k \in\left(0, k^{\star}\right)$ and $\left\|\tilde{\vartheta}^{\eta}\right\| \leq \rho^{\star}$.

The estimator-based switching scheme consists in monitoring the performance indexes at each instant. After a switch has occurred, a period called waiting time of length $\tau_{\min }$ is allowed to elapse before the next switch can take place. The waiting time is essential to prevent arbitrarily fast switching. Note that the waiting time for the dwell-time switching is equal to $\tau_{D}$, whereas for the hysteresis switching the waiting time is proportional to the hysteresis constant $h$. The following theorem establishes the stability properties of the closed-loop system with estimator-based switching logic:

Theorem 4.5: For the system (17) and the switching logic described in Section III-B, there exist positive numbers $\tau_{s}, k^{\star}, \rho^{\star}$ and a function $\rho_{s}\left(\mu, \tau_{\min }\right)>0$ such that if the waiting time $\tau_{\min }$ satisfies $\tau_{\min } \in\left(0, \tau_{s}\right)$ and $\rho \leq$ $\min \left\{\rho^{\star}, \rho_{s}\left(\mu, \tau_{\min }\right)\right\}$, then the trajectories of the closedloop system originating from any initial conditions $x_{0} \in \mathbb{R}^{r}$, $\omega_{0} \in \mathbb{R}^{2}, \hat{\omega}_{0} \in \mathbb{R}^{2}$ and $\hat{\zeta}_{0} \in \mathbb{R}^{2}$ are bounded and satisfy $\lim _{t \rightarrow \infty} y(t)=0$ for all $\mu \in \mathcal{P}$ and $k \in\left(0, k^{\star}\right)$.

The proof is carried out by contradiction and can be found in [19]. Given $\tau_{s}$, one can accordingly choose the waiting time $\tau_{\text {min }}$, then select $\tau_{D}=\tau_{\min }$ for the dwell-time logic and a sufficiently small $h$ for the hysteresis switching such that the condition $\tau_{\min } \in\left(0, \tau_{s}\right)$ is verified.

\section{ILlustrative EXAMPLE}

In this section, we provide a simulation study to show the effectiveness of the proposed algorithms and com-
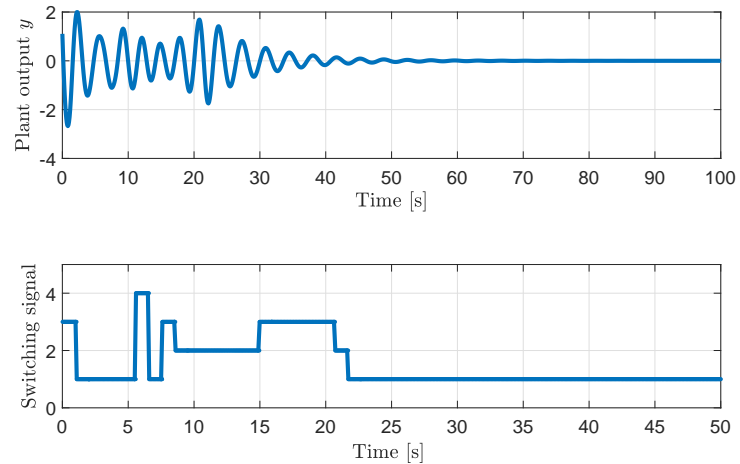

Fig. 9. System response using the dwell-time switching mechanism
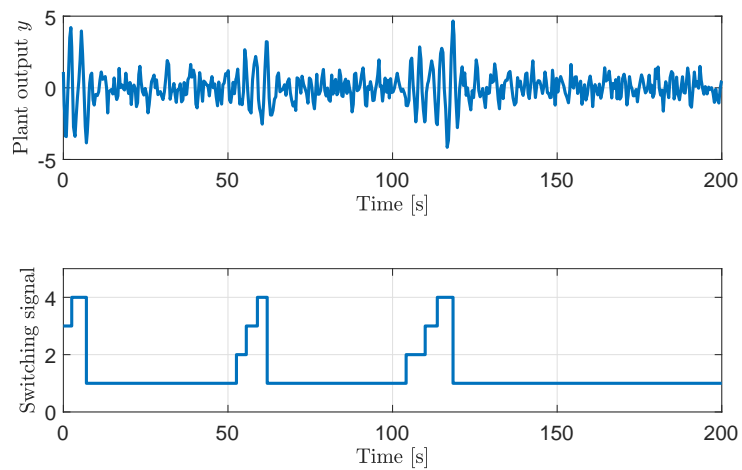

Fig. 10. System response with additional input noise: Pre-routed switching

pare the performance of the three different switching logics. Consider the stable non-minimum phase plant model $W(s)=\frac{2(s-1)}{s^{2}+2 s+5}$, and let the disturbance signal be given by $d(t)=2 \sin (2 t)$. The frequency response of the plant at the frequency of excitation yields the parameter vector $\theta=(0.8235,-0.7059)^{T}$. The family of the candidate controllers are chosen as $\mathcal{I}:=\{1,2,3,4\}$ with corresponding parameter estimates : $\hat{\theta}^{1}=(1,0)^{T}, \hat{\theta}^{2}=(0,-1)^{T}, \hat{\theta}^{3}=$ $(-1,0)^{T}, \hat{\theta}^{4}=(0,1)^{T}$. Note that the considered family corresponds to four different controllers of the type proposed in [13]. To examine the worst case scenario, we choose the third controller as the initial controller, whose parameter estimate is located at the furthest location from the true value. The gain parameters for all three switching mechanisms are $k=0.5, \tau_{D}=1, h=0.35, \epsilon=0.1, \lambda=1 e-3$.

The time history of the plant output and that of the switching signal are reported in Fig. 7 through Fig. 9 for each of the three switching logic. It is observed that the proposed multiple controller with all three switching mechanisms succeed in cancelling the periodic disturbance with comparable convergence speed and transient behavior. It is seen that each switching logic is capable of selecting the most appropriate controller $\left(\hat{\theta}_{1}\right.$ in this case). It is noted that the hysteresis switching approach can be tuned to achieve a faster response; however, the tuning parameters have been purposefully selected to enforce a similar convergence speed for all three systems to facilitate a comparative analysis.

Next, the robustness of the switching mechanisms is tested by adding additive noise at the plant input. Specifically, the sinusoidal disturbance is replaced by the signal $d(t)=$ 

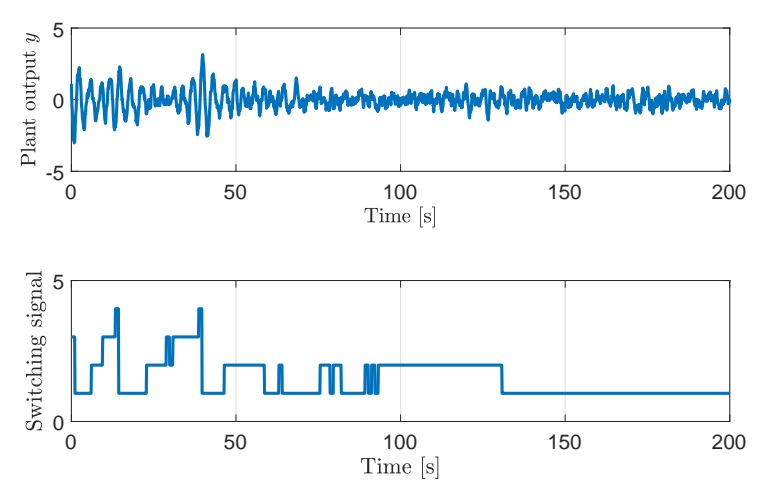

Fig. 11. System response with additional input noise: Dwell-time switching
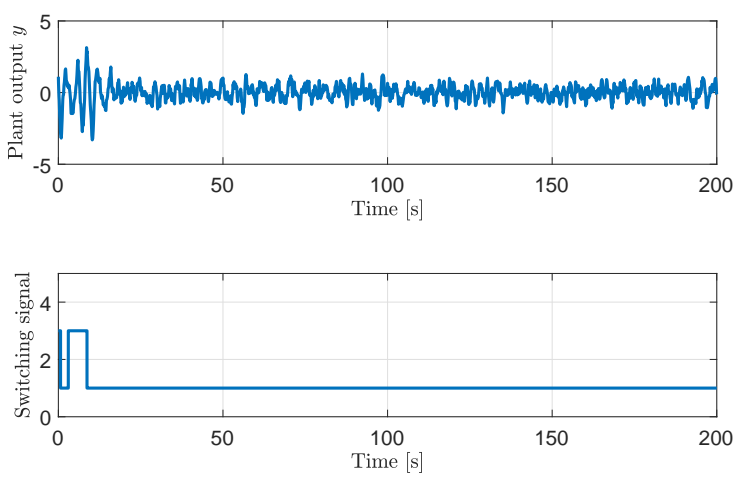

Fig. 12. System response with additional input noise: Hysteresis switching

$2 \sin (2 t)+n(t)$, where $n(t)$ is uniformly distributed in $[-2,2]$. Note that this is a stringent test, as the magnitude of the noise is comparable to that of the sinusoidal signal. The same selection of controller parameters has been adopted for the simulations. As seen in Fig. 10 and Fig. 11, the behavior the pre-routed and dwell-time switching controller has worsened considerably due to the effect of the input noise, especially for the pre-routed scheme. On the other hand, the hysteresis-based switching method demonstrates better robustness properties.

\section{CONCLUding REMARKS}

A state-sharing multi-controller architecture has been proposed to overcome the necessity of knowing the parameters related to the frequency response of the stabilized plant (or their sign) in the adaptive feedforward approach to the harmonic disturbance rejection problem. The rationale behind the method is to design a sufficient large number of multiple model-based controllers such that there exist models with constant parameter estimates that are sufficiently close to the unknown parameter vector of the plant. Then, using a suitable performance criterion and proper switching logic, one can eventually find the most appropriate model, and consequently activate the corresponding controller.

The obvious drawback of the algorithm is its low efficiency due to the possible high dimension of the multiple models, notwithstanding the reduction allowed by using a state-shared technique. While in the single harmonic case the order of these controllers compares very favorably with the order of the multiple-model adaptive controller proposed in [14], this advantage disappears quickly in a more general case when multiple harmonics are considered. At this point it is unclear whether the switching strategies proposed here are advantageous from the dimensionality standpoint versus a multi-frequency version of the adaptive controller proposed in [14]. It appears that a judicious combination of the method proposed in this paper with the multiple-model adaptive control of [14] is perhaps the winning strategy to obtain a "universal" adaptive feedforward regulator with the smallest possible dimension. This avenue is currently being pursed and extended to the case of multiple harmonics.

\section{REFERENCES}

[1] K. Ariyur and M. Krstic, "Feedback attenuation and adaptive cancellation of blade vortex interaction on a helicopter blade element," IEEE Transactions on Control Systems Technology, vol. 7, no. 5, pp. 596-605, 2002.

[2] S. M. Kuo and D. R. Morgan, "Active noise control: A tutorial review," Proceedings of the IEEE, vol. 87, no. 6, pp. 943-973, 1999.

[3] H. I. Basturk and M. Krstic, "Adaptive wave cancelation by acceleration feedback for ramp-connected air cushion-actuated surface effect ships," Automatica, vol. 49, no. 9, pp. 2591 - 2602, 2013.

[4] B. Francis and W. Wonham, "The internal model principle of control theory," Automatica, vol. 12, no. 5, pp. 457 - 465, 1976.

[5] B. A. Francis, "The linear multivariable regulator problem," SIAM Journal on Control and Optimization, vol. 15, no. 3, pp. 486-505, 1977.

[6] E. Davison, "The robust control of a servomechanism problem for linear time-invariant multivariable systems," IEEE Transactions on Automatic Control, vol. 21, no. 1, pp. 25-34, Feb 1976.

[7] B. A. Francis and W. M. Wonham, "The role of transmission zeros in linear multivariable regulators," International Journal of Control, vol. 22, no. 5, pp. 657-681, nov 1975.

[8] M. Bodson, A. Sacks, and P. Khosla, "Harmonic generation in adaptive feedforward cancellation schemes," IEEE Transactions on Automatic Control, vol. 39, no. 9, pp. 1939-1944, 1994.

[9] W. Messner and M. Bodson, "Design of adaptive feedforward algorithms using internal model equivalence," International Journal of Adaptive Control and Signal Processing, vol. 9, no. 2, pp. 199-212, 1995.

[10] M. Bodson, J. Jensen, and S. Douglas, "Active noise control for periodic disturbances," IEEE Transactions on Control Systems Technology, vol. 9, no. 1, pp. 200-205, oct 2001.

[11] J. Chandrasekar, L. Liu, D. Patt, P. Friedmann, and D. Bernstein, "Adaptive Harmonic Steady-State Control for Disturbance Rejection," IEEE Transactions on Control Systems Technology, vol. 14, no. 6, pp. 993-1007, nov 2006.

[12] S. Pigg and M. Bodson, "Adaptive Algorithms for the Rejection of Sinusoidal Disturbances Acting on Unknown Plants," IEEE Transactions on Control Systems Technology, vol. 18, no. 4, pp. 822-836, 2010.

[13] R. Marino and P. Tomei, "Output Regulation for Unknown Stable Systems," IEEE Transactions on Automatic Control, vol. 60, no. 8, pp. 2213-2218, 2015.

[14] Y. Wang, G. Pin, A. Serrani, and T. Parisini, "Removing SPR-like conditions in adaptive feedforward control of uncertain systems," in Proceedings of the IEEE Conference on Decision and Control, Las Vegas, NV, 2016, pp. 4728-4733.

[15] A. S. Morse, "Control using logic-based switching," in Trends in Control. A European Perspective. Springer Verlag, 1995, pp. 69113.

[16] S. Fujii, J. P. Hespanha, and A. S. Morse, "Supervisory control of families of noise suppressing controllers," in Proceedings of the 37th IEEE Conference on Decision and Control, vol. 2, 1998, pp. 16411646.

[17] J. P. Hespanha, D. Liberzon, and A. Morse, "Hysteresis-based switching algorithms for supervisory control of uncertain systems," Automatica, vol. 39, no. 2, pp. $263-272,2003$.

[18] M. Cao and A. S. Morse, "Dwell-time switching," Systems and Control Letters, vol. 59, no. 1, pp. 57-65, 2010.

[19] K. Narendra and J. Balakrishnan, "Adaptive control using multiple models," IEEE Transactions on Automatic Control, vol. 42, no. 2, pp. 171-187, 1997. 